\title{
Challenges with engaging participants in behavioral intervention research trials
}

This article was published in the following Dove Press journal:

Open Access Journal of Clinical Trials

17 November 2009

Number of times this article has been viewed

\section{David Olem \\ Kelly M Sharp \\ Mallory O Johnson}

Department of Medicine, University of California, San Francisco, CA, USA
Correspondence: Mallory O Johnson Department of Medicine, University of California, San Francisco,

50 Beale Street, Suite 1300 ,

San Francisco, CA 94105, USA

$\mathrm{Tel}+\mathrm{I}(415)$ 597-9374

Fax +I (4I5) 597-9340

Email mallory.johnson@ucsf.edu
Abstract: In clinical trials research, nonadherence to an investigational health promotion intervention poses threats to internal validity, thus raising concern over the trial outcomes and interpretation of results. Successfully helping participants to engage in and complete a multisession intervention can be particularly challenging. This task can become more complicated when working with participants who are dealing with chronic health conditions and are from diverse cultural, socio-economic, and marginalized backgrounds who may have competing needs, beliefs, and priorities that conflict with the needs of the trial protocols. This paper identifies some of the challenges to helping individuals participate in the sessions of a behavioral intervention and shares a set of strategies developed by a clinical research team to engage with participants and minimize barriers to completing the intervention.

Keywords: clinical trials, behavioral interventions, retention, internal validity

The establishment of evidence for innovative health interventions requires careful evaluation, often employing randomized controlled trial (RCT) designs. ${ }^{1-4}$ Methodological standards are rigorous for biomedical interventions such as pharmacotherapies as well as for behavioral programs to promote health, prevent disease, or avert complications from disease. Among threats to internal validity is the risk from nonadherence to the investigational intervention, as nonadherence within a trial can deem results difficult to interpret. ${ }^{5}$ Negative findings may indicate a lack of efficacy for the intervention or may be an artifact of poor participation or adherence within the trial..$^{6-11}$ For example, in multi-site clinical trials, variability in protocol compliance or study retention by site can result in analytic challenges that limit the impact of the trial results. ${ }^{9}$ The purpose of this paper is to share lessons learned from the conduct of behavioral counseling interventions within clinical research settings. While factors that influence overall retention and attrition in a trial may overlap with engagement in the intervention, ${ }^{12-15}$ we focus specifically on participation in intervention components of trials testing behavioral interventions.

Our observations are informed by our experience in conducting several randomized controlled trials of behavioral interventions. These studies evaluate interventions designed to address issues of coping with medical treatment, treatment decision-making, and adherence to treatment plans with HIV-positive adult men and women. In particular, we draw examples from a recent RCT (registration number NCT00643903) of a behavioral intervention to improve coping with treatment side effects. In this trial, a convenience sample of $250 \mathrm{HIV}$-positive adults taking antiretroviral therapy were recruited from the community and randomized to the behavioral intervention or a wait list control. 
The intervention consisted of 5 one-on-one sessions, which were 60 to 90 minutes long. Each session consisted of a brief check-in, instruction and practice of specific skills, which included communication, support utilization, coping effectiveness, and problem solving around concerns about HIV treatment and side effects. Intervention counselors/facilitators encouraged participants to set goals at the end of each session, which were used as a strategy to provide continuity between sessions. Participants were incentivized for completing all five sessions within the specified timeline with a US\$30 payment, which they received at a subsequent follow up assessment. The incentive payment had varied effects on participants' motivation; while some participants appeared to be motivated by the incentive, others reported that they felt underpaid or noted that other studies paid more, and still others reported that the payment did not affect their decision to participate.

Through the conduct of this and other behavioral intervention RCTs, we have identified several recurring and common challenges to engaging our participants in the study, and we have identified key strategies for initiating engagement and maintaining adherence throughout the study. Of the 128 participants randomized to receive the intervention described above, $116(90.6 \%)$ completed at least 1 session and, of those who had 1 session, 112 (96.5\%) completed all 5 sessions. These rates of intervention participation reflect an improvement from our prior studies, in which we did not systematically address the challenges described in this paper. The challenges and recommendations to overcome them are described below.

\section{Challenges}

There are multiple barriers to fully engaging participants in this behavioral intervention. The challenges begin with persuading participants to show up for the first intervention session and extend to efforts to encourage them to actively participate in the content of each session. While some participants report being motivated to attend the intervention sessions and report a belief that he/she would benefit from the information and support provided, a sizable number present the following practical and motivational challenges to successful intervention engagement and completion. The list below is based on investigator and staff observation and direct participant feedback.

\section{The participant never intended}

\section{to attend intervention sessions}

The participant may have thought that he/she had to pretend to be interested in participating in the intervention in order to get paid for the assessment, or may have hoped to be randomized to a condition that did not involve intensive participation. The participant may have just focused on getting the payment from the assessment for a specific and immediate need such as paying rent. Even after completing the first intervention session, the participant may continue to struggle with attendance to the remainder of the intervention sessions.

\section{Fear of emotional interaction}

Participants may be concerned about the potential pressure to discuss emotionally charged topics. A participant may have learned to cope by avoiding stressful or emotionally charged material, particularly if the participant has a history of trauma or abuse. Avoidance may have been an effective coping strategy in their prior experience.

\section{Uncertain benefit}

Some participants report that they are currently connected to multiple resources and feel the intervention will either be repetitive or take up time without much gain.

\section{Chaotic life situations}

Participants may experience turmoil due to mental health issues, cognitive impairments, psychosocial stressors, economic instability, substance use or a combination of these factors. The participants' life circumstances may change from day to day, making it difficult to maintain their ongoing participation in the intervention.

\section{Health problems}

Many participants encounter challenges with known or unexpected medical situations that impede participation. These included hospitalizations, last minute doctor's appointments and medication side effects such as nausea and diarrhea. These health complications may come on suddenly and may result in a no-show or reschedule of the intervention session.

\section{Contact difficulties}

The participant may have no phone, or have limited contact information.

\section{Recommendations}

We have developed a set of engagement strategies with participants in efforts to increase adherence and completion of investigational interventions. The strategies were developed over time through feedback from participants, 
input from community partners and advisory boards, as well as clinical supervision which included case presentations and brainstorming sessions. The challenges often co-occur and the following recommendations may be applied to several challenges. We recognize that structure and available resources impact the ability to deliver the strategies we identified and recommend that a broad and flexible approach be considered when exploring methods to engage participants.

\section{Minimize breaks in contact}

Facilitators should contact participants quickly following assignment to an experimental intervention condition. Ideally, the participant meets the facilitator at the appointment in which he/she is randomized. This can alleviate concern of not knowing who the counselor will be and the first session can be scheduled as soon as possible. Asking the participant's permission for reminders (and preference for timing and format of reminders) can engage the participant in a collaborative process.

\section{Explore potential barriers to participation}

In the initial contact, the facilitator should ask the participant "Is there anything that could make it hard for you to get to your appointments?" If the participant identifies any barriers such as "It's hard for me to get up in the morning" the facilitator engages in problem-solving with the participant around that specific barrier. This also gives the facilitator a chance to explore how this barrier to attending intervention sessions may be impacting other areas of the participant's life. For example, is not being able to get up in the morning causing the participant to miss other commitments such as doctors' appointments or job training classes? If so, the facilitator should reinforce any efforts from the participant to engage in the intervention, such as acknowledging when a participant calls to reschedule rather than just not showing up. Reframing in this way allows the participant to see they have made some progress around the initial barrier and can also help the facilitator connect with the participant.

\section{Use clinical skills to keep participant engaged}

Using clinical judgment, facilitators should assess ambivalence and resistance throughout the intervention and remain client-centered by using the participant's real life challenges as links back to the intervention. For example, facilitators might say "It sounds like you have a lot going on and these sessions aren't a priority. I wonder if we can focus our time together on problem solving solutions to your current situation." Facilitators can continue to demonstrate their flexibility and understanding by rolling with the resistance, using open-ended questions, affirmations and reflective listening. In an effort to "roll with the resistance" a facilitator might say "I really hear that you're upset about having to arrange your day around this appointment, and I appreciate you taking the time to be here. I will do my best to keep us on track and let me know if there is anything I can do to make this work best for you." By not tackling the participant's anger directly, the facilitator acknowledges the participant's feelings and conveys that he/she wants to work with their goals, not against them. This serves to diffuse the participant around a potential barrier and allows them to engage in the intervention content. Later in the session, the facilitator may be able to refer back to the participant's initial irritation and explore ways that communication in this example served to contribute to or inhibit the participant from getting their needs met. This provides another opportunity for the facilitator to reframe the initial irritation in terms of effective communication.

\section{Make the participant feel respected and appeal to his/her desire to help out with study}

Often participants enroll in studies out of a desire to help others through their involvement or sharing of their experiences, challenges, and successes. Reminding the participant how important such contributions are to the research can serve as a positive motivator to complete the sessions. For instance, many people with HIV have experienced stigma and marginalization, and have been treated as if they have nothing important to contribute. By reinforcing the importance of learning about the participant's experiences and how their study participation may be helpful to others, facilitators validate these motivations, which will likely result in greater intervention participation.

\section{Be flexible and accommodating}

Participants' lives may be stressful or chaotic, and may contain competing priorities, making a rigid session schedule unrealistic. A key to successful engagement is flexibility, allowing built-in time for the participant to be late and making efforts to be understanding. Repeated participant no-shows may be frustrating, and such irritation could potentially come through in the facilitator's tone of voice and could damage the relationship, especially if the participant was already experiencing life stress and possible shame around having missed an appointment. Using clinical supervision which includes listening to recordings of sessions and giving feedback, as well 
as both formal and informal consultation with colleagues, is critical to keeping facilitator emotions from affecting the likelihood of the participant's continued involvement.

\section{Use varying contact strategies: emails/letters/texting}

Catching the participant at the right time and by the right modality can make a big difference. The participant may have been distracted when listening to a phone message or may have been meaning to call back, but has been preoccupied with other matters. More recently, some participants have reported that they do not check voicemail but do respond to text messages. Keeping in touch shows the participant that he/she is wanted in the study. If a participant doesn't respond to phone messages, emails and letters can be sent, assuming consent has been given for such modes of contact. A letter or email might read, "We have been unable to contact you by phone. Your participation in the study is very important to us. Please let us know how we can make your attendance as convenient as possible." Maintaining supportive persistence without putting unnecessary pressure on the participant is important. Using supervisors and team members for advice about the frequency, mode, and tone of communications to participants is helpful.

\section{Be a good host}

People remember when they have been treated well or made to feel at home. Conversely, people remember when they have been treated poorly or made to feel unwelcome, particularly if they have experienced stigma and discrimination in other parts of their lives. Treating participants with respect, offering gestures of hospitality such as healthy snacks, coffee or tea, and reinforcing the study's appreciation of their contribution can increase engagement with the intervention and can provide a corrective positive experience that can counteract previous negative occurrences.

\section{Build connections to the community}

People may feel more comfortable getting services from a place they know, and going outside of their trusted community to discuss personal matters can cause anxiety. Participants, especially those from marginalized populations and at the greatest risk for negative health outcomes, might wonder if they are going to be treated with respect and without judgment. Recommendations from trusted providers within someone's community can go a long way. Building collaborative relationships with local agencies and providers is a helpful way to gain credibility within the community of interest. ${ }^{16}$

\section{Assure participant feels a sense of progress in each session}

There may be mandated content to cover in each session and the first session may require gathering information about the participant. The facilitator should reserve time to address issues that appear relevant to the participant. Active problem solving can help the participant identify a clear plan for dealing with specific issues related to the intervention or to the participant's ability to actively participate. The participant then is more likely to leave the session with a sense that tangible progress has been made, resulting in relief around something that was initially stressful. For example, a participant may identify that noisy neighbors are affecting her quality of sleep, which in turn makes it hard for her to keep appointments. It may be helpful to generate potential solutions to dealing with the neighbors and to model problem solving around the issue. Such interactions may serve to increase participants' investment in the intervention while also providing a tangible sense of progress.

\section{Summary and conclusions}

The challenges and recommendations outlined in this paper are informed by clinical trials of behavioral interventions in the context of HIV-infected populations. Although the specific nature of this trial may restrict the generalizability of the recommendations to other populations, illnesses, trial designs and intervention modalities, the general concepts are likely applicable across settings and are consistent with the limited literature on this topic. ${ }^{8,13}$ For example, there is a documented need to address expectations, motivations, and barriers to clinical trial participation across gender, race and ethnicity. $12,13,16,17$ The few sources that address these issues often do so in the context of engagement in treatment rather than clinical trials, ${ }^{18,19}$ and while there may be some attention to the needs of special populations such as homeless persons, ${ }^{20}$ this literature typically fails to consider issues unique to a research setting such as altruism and desire for financial incentives. ${ }^{17}$ Without effective techniques to maximize adherence to innovative health promotion interventions, investigators will struggle to compile empirical evidence of the intervention's efficacy and eventual effectiveness.

\section{Acknowledgments}

This work was supported grants R01MH068208 and K24MH087220 from the National Institutes of Health.

\section{Disclosures}

The authors disclose no conflicts of interest. 


\section{References}

1. Boutron I, Moher D, Altman DG, Schulz KF, Ravaud P. Methods and processes of the CONSORT Group: example of an extension for trials assessing nonpharmacologic treatments. Ann Intern Med. 2008;148(4): W60-W66.

2. Kane RL, Wang J, Garrard J. Reporting in randomized clinical trials improved after adoption of the CONSORT statement. J Clin Epidemiol. 2007;60(3):241-249.

3. Peto R, Collins R, Gray R. Large-scale randomized evidence: large, simple trials and overviews of trials. J Clin Epidemiol. 1995;48(1):23-40.

4. Nezu AM, Nezu CM. Evidence-Based Outcome Research: A Practical Guide to Conducting Randomized Controlled Trials for Psychosocial Interventions. New York, NY, US: Oxford University Press; 2008:486.

5. Walsh JC. Adherence in clinical trials and in clinical practice. Antivir Ther. 1999;4 Suppl 3:49-54.

6. Johnson MO, Remien RH. Adherence to research protocols in a clinical context: Challenges and recommendations from behavioral intervention trials. Am J Psychother. 2003;57(3):348-360.

7. Gossec L, Tubach F, Dougados M, Ravaud P. Reporting of adherence to medication in recent randomized controlled trials of 6 chronic diseases: a systematic literature review. Am J Med Sci. 2007;334(4):248-254.

8. Shumaker SA, Dugan E, Bowen DJ. Enhancing adherence in randomized controlled clinical trials. Control Clin Trials. 2000; 21(5 Suppl):226S-232S.

9. Land SR, Ritter MW, Costantino JP, et al. Compliance with patientreported outcomes in multicenter clinical trials: methodologic and practical approaches. J Clin Oncol. 2007;25(32):5113-5120.

10. Lui KJ. Correcting for non-compliance of repeated binary outcomes in randomized clinical trials: randomized analysis approach by Yutaka Matsuyama. Statistics in Medicine 2002;21:675-687. Stat Med. 2007;26(25):4679-4683.
11. Bang H, Davis CE. On estimating treatment effects under non-compliance in randomized clinical trials: are intent-to-treat or instrumental variables analyses perfect solutions? Stat Med. 2007;26(5):954-964.

12. Milligan CO, Nich C, Carroll KM. Ethnic differences in substance abuse treatment retention, compliance, and outcome from two clinical trials. Psychiatr Serv. 2004;55(2):167-173.

13. Wilcox S, Shumaker SA, Bowen DJ, et al. Promoting adherence and retention to clinical trials in special populations: a women's health initiative workshop. Control Clin Trials. 2001;22(3):279-289.

14. Johnson MO, Dilworth SE, Neilands TB, et al. Predictors of attrition among high risk HIV-infected participants enrolled in a multi-site prevention trial. AIDS Behav. 2008;12(6):974-977.

15. Leonard NR, Lester P, Rotheram-Borus MJ, Mattes K, Gwadz M, Ferns B. Successful recruitment and retention of participants in longitudinal behavioral research. AIDS Educ Prev. 2003;15(3):269-281.

16. Bogart LM, Uyeda K. Community-based participatory research: partnering with communities for effective and sustainable behavioral health interventions. Health Psychol. 2009;28(4):391-393.

17. Rosenbaum JR, Wells CK, Viscoli CM, Brass LM, Kernan WN, Horwitz RI. Altruism as a reason for participation in clinical trials was independently associated with adherence. $J$ Clin Epidemiol. 2005;58(11):1109-1114.

18. Mugavero MJ. Improving engagement in HIV care: what can we do? Top HIV Med. 2008;16(5):156-161.

19. Kurella Tamura M, Goldstein MK, Perez-Stable EJ. Preferences for dialysis withdrawal and engagement in advance care planning within a diverse sample of dialysis patients. Nephrol Dial Transplant. In press. 2009

20. Conanan B, London K, Martinez L, et al. Adapting Your Practice: Treatment and Recommendations for Homeless Patients with HIV/AIDS. Nashville, TN: National Health Care for the Homeless Council, Inc; 2003.
Open Access Journal of Clinical Trials

\section{Publish your work in this journal}

The Open Access Journal of Clinical Trials is an international, peerreviewed, open access journal publishing original research, reports, editorials, reviews and commentaries on all aspects of clinical trial design, management, legal, ethical and regulatory issues, case record form design, data collection, quality assurance and data auditing

\section{Dovepress}

methodologies. The manuscript management system is completely online and includes a very quick and fair peer-review system, which is all easy to use. Visit http://www.dovepress.com/testimonials.php to read real quotes from published authors. 